\title{
Internal Iliac Pseudoaneurysm Rupture Attributed to Perforated Hartmann's Pouch Diagnosed on CT
}

\author{
Jeremy R. Camden, Laura R. Carucci \\ Department of Radiology, Virginia Commonwealth University, Richmond, USA \\ Email: camdenjr@gmail.com
}

Received May 5, 2012; revised June 1, 2012; accepted June 8, 2012

\begin{abstract}
Rupture is a rare but disastrous complication of an arterial aneurysm. Intraabdominal abscess in association with iliac artery aneurysm is uncommon but can predispose to aneurysm rupture. We report the Computed Tomography (CT) findings of an internal iliac artery pseudoaneurysm formed by a pelvic abscess attributed to the perforation of the patient's Hartmann's pouch. Rupture of the pseudoaneurysm resulted in the patient's death.
\end{abstract}

Keywords: Iliac Artery Pseudoaneurysm; Hartmann’s Pouch; Rupture; Computed Tomography

\section{Introduction}

The Society of Vascular Surgery and the North American Chapter of the International Society of Cardiovascular Surgery defines an aneurysm as a permanent localized dilation of an artery with a diameter greater than $50 \%$ of the expected size [1]. However, pseudoaneurysms result from an arterial leak contained by the surrounding tissues and do not contain an arterial wall. Most pseudoaneurysms are caused by trauma. Aneurysms of the iliac arteries are uncommon but dangerous because they often remain asymptomatic until they rupture [2]. Infected true aneurysms and pseudoaneurysms merit special consideration because of the increased propensity to rupture. Imaging may detect these aneurysms/pseudoaneurysms incidentally and detection prior to rupture is critical.

\section{Case Report}

A 40-year-old female with past medical history significant for metastatic colon cancer status post sigmoidectomy and diverting colostomy at an outside hospital presented to our institution after having rectal pain and bright red blood per rectum (BRBPR). There was no blood near the patient's colostomy. The patient had an additional history of left nephrectomy, right-sided obstructive uropathy with nephrostomy placement, hysterectomy and bilateral salpingo-oophorectomy, hepatic wedge resection, multiple hepatic arterial chemoembolizations for metastatic disease, and deep venous thrombosis with inferior vena cava filter placement.

In the emergency room, the patient was hypotensive and tachycardic with hemoglobin $4.0 \mathrm{~g} / \mathrm{dL}$ (critical low 7.1). The patient was resuscitated with 2 units of packed red blood cells and 4 liters of normal saline intravenously and admitted to the medical intensive care unit. The patient further decompensated after an episode of large BRBPR and an emergent abdominal-pelvic CT exam was performed.

On contrast enhanced CT, an abscess in the pelvis measuring $7.5 \times 5.7 \times 7.9 \mathrm{~cm}$ was identified. The abscess surrounded a smaller vascular outpouching measuring $1.8 \times 1.8 \times 3.4 \mathrm{~cm}$ communicating with the left internal iliac artery, consistent with a pseudoaneurysm (Figures 1(a) and (b)). A small locule of nondependent air within the irregularly shaped vascular nidus suggested perforation and communication with the surrounding abscess containing the pseudoaneurysm (Figure 1(c)). The left internal iliac pseudoaneurysm was directly contiguous with an irregular Hartmann's pouch suture line. There was disruption of the Hartmann's pouch wall with an adjacent collection of complex fluid and air interposed between the Hartmann's pouch and the pseudoaneurysm (Figure 1(d)). Breakdown of the Hartmann's pouch suture line and blood clot within the Hartmann's pouch were noted. Small amorphous appearing hyperdensities with attenuation similar to the vascular nidus within the Hartmann's pouch were observed, consistent with pooling of active extravasation from the pseudoaneurysm (Figure 1(e)).

While the patient was in the CT room, she had another episode of large BRBPR and became unresponsive and pulseless. A code was activated and advanced cardiopulmonary resuscitation was performed for 19 minutes, but the patient succumbed. The cause of death was attributed to rupture of the left internal iliac pseudoaneurysm. 


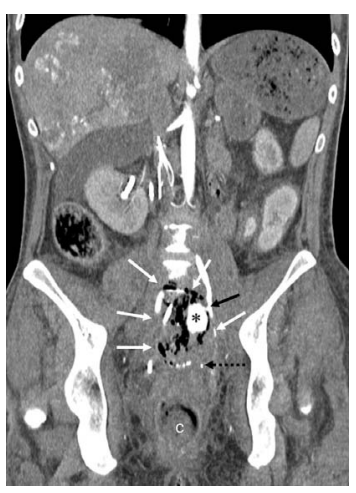

(a)

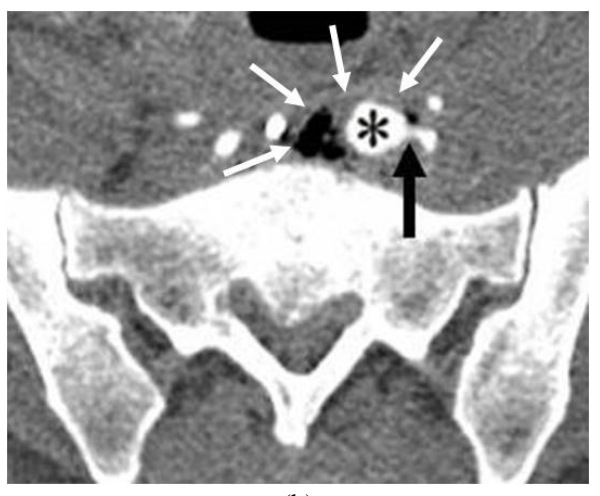

(b)

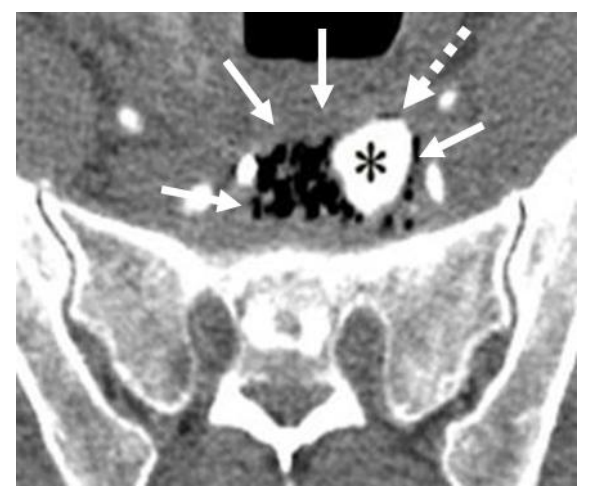

(c)

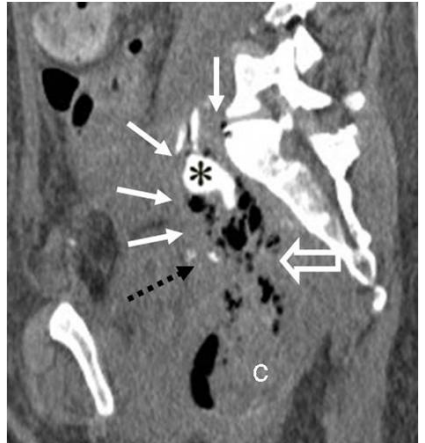

(d)

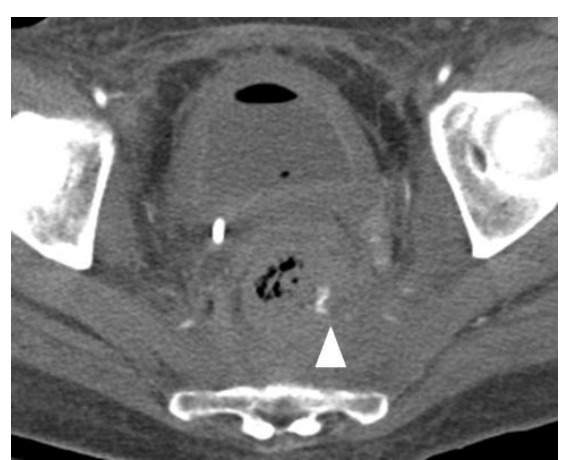

(e)

Figure 1. Internal iliac pseudoaneursym formed by abscess due to Hartmann's pouch leak in a 40 year-old female with history of colon cancer; (a) Contrast enhanced coronal CT image shows an abscess measuring $7.5 \times 5.7 \times 7.9 \mathrm{~cm}$ within the pelvis (white arrows) surrounding a vascular outpouching $\left(^{*}\right)$ measuring $1.8 \times 1.8 \times 3.4 \mathrm{~cm}$ and communicating with the left internal iliac artery by a narrow neck (black arrow), consistent with pseudoaneursym. The left internal iliac pseudoaneurysm is directly contiguous with an irregular Hartmann's pouch suture line (dashed black arrow). Blood clot (c) within the Hartmann's pouch is shown. Hepatic metastases, ascites, and inferior vena cava filter are also shown; (b) Axial CT image depicts the vascular nidus $\left(^{*}\right)$ communicating with the left internal iliac artery by a narrow neck (black arrow). Abscess (white arrows) forming the pseudoaneurysm is displayed; (c) Axial CT image slightly caudal to B demonstrates a small locule of nondependent air (dashed white arrow) within the irregularly shaped vascular nidus (*) of the left internal iliac pseudoaneurysm with surrounding abscess (white arrows), which suggests perforation; (d) Sagittal oblique image at the level of the vascular nidus (*) displays the irregular contour of the nidus $(*)$ and the surrounding abscess (white arrows). There is disruption of the Hartmann's pouch suture line (dashed black arrow) with a collection of complex fluid and air communicating between the Hartmann's pouch and the pseudoaneurysm (open white arrow). Blood clot (c) within the Hartmann's pouch is also depicted; (e) Axial CT image at the level of the Hartmann's pouch shows amorphous hyperdensity (white arrow head) surrounded by complex fluid and air within the Hartmann's pouch, consistent with pooling of active extravasation from the pseudoaneurysm. Air within the urinary bladder related to recent Foley catheterization is also noted.

\section{Discussion}

The Hartmann procedure is a relatively common surgery in which a diverting end-colostomy is created and the rectal stump is closed with suture (Hartmann's pouch). This procedure may be performed for resection of a rectosigmoid carcinoma, complicated diverticulitis, or penetrating trauma [3]. Possible rare life threatening complications of the Hartmann procedure include leakage or even blowout of the blind-ending rectal or colonic stump $[3,4]$. Leak from the Hartmann's pouch often leads to abscess but this complication may be managed conservatively due to diversion of the fecal stream. However, this case report is the first, to our knowledge, to demonstrate diagnostic CT images of an internal iliac artery pseudo- aneurysm attributed to Hartmann's pouch perforation, which ruptured causing exsanguination.

Although lower gastrointestinal bleeding related to iliac artery aneurysm rupture or fistula formation into bowel has been previously reported, this case is different because the patient had a Hartmann's pouch leak causing abscess and resulting in pseudoaneurysm formation [5-7]. This case report emphasizes the role of CT in diagnosis of iliac pseudoaneurysms. Diagnosis before rupture is essential for patient survival, especially in association with infection.

Persistent rectal bleeding in a patient with a Hartmann's pouch suggests the possibility of a surgical complication and may warrant an urgent contrast enhanced CT to evaluate for the source of bleeding. Careful as- 
sessment for iliac artery irregularity should be made on CT in a patient bleeding from a Hartmann's pouch. The Hartmann's pouch suture line should also be evaluated. Coronal and sagittal reformatted images may aide in evaluation of the Hartmann's pouch and the vasculature. In this case, the insidious onset of rectal bleeding was likely due to pseudoaneurysm leakage rather than rupture. Herald bleeding may allow time for resuscitation and diagnosis. Locules of air within the vascular nidus of a pseudoaneurysm as demonstrated in this case suggest perforation and source of bleeding. Contrast enhanced CT could be helpful in the early diagnosis of pseudoaneurysms formed by abscess and should alert the radiologist and clinician that urgent treatment is necessary prior to impending life threatening rupture.

\section{REFERENCES}

[1] K. W. Johnston, R. B. Rutherford, M. D. Tilson, et al., "Suggested Standards for Reporting on Arterial Aneurysm. Subcommittee on Reporting Standards for Arterial Aneurysm, Ad Hoc Committee on Reporting Standards, Society for Vascular Surgery and North American Chapter, International Society for Cardiovascular Surgery," Jour- nal of Vascular Surgery, Vol. 13, No. 3, 1991, pp. $452-$ 458. doi:10.1067/mva.1991.26737

[2] P. W. Zimmer, E. J. Raker and T. M. Quigley, "Isolated Hypogastric Artery Aneurysms,” Annals of Vascular Surgery, Vol. 13, No. 5, 1999, pp. 545-549. doi:10.1007/s100169900296

[3] F. C Bakker, H. F. W. Hoitsma and G. Den Otter, "The Hartmann Procedure,” British Journal of Surgery, Vol. 69, No. 10, 1982, pp. 580-582. doi:10.1002/bjs.1800691007

[4] E. J. C. Lubbers and H. M. M. de Boer, "Inherent Complications of Hartmann's Operation,” Surg Gynecol Obstet, Vol. 155, No. 5, 1982, pp. 717-721.

[5] J. Katoh, S. Shindo, S. Kina, et al., "Rupture of an Isolated Internal Iliac Artery Aneurysm into the Rectum: Report of a Case,” Surgery Today, Vol. 25, No. 6, 1995, pp. 554556. doi:10.1007/BF00311315

[6] H. M. Noh, E. K.Fishman and D. A.Bluemke, "Isolated Common Iliac Artery Aneurysm Rupturing into Small Bowel: CT Appearance,” Abdominal Imaging, Vol. 20, No. 6, 1995, pp. 521-522. doi:10.1007/BF01256703

[7] A. Ingu, N. Takagi, O.Izumiyama, et al. "Isolated Iliac Artery Aneursysmocolonic Fistula with Pericolic Abscess,” Ann Vasc Surg, Vol. 202, No. 16, pp. 663-665. 